\title{
Communication \\ Improving Calibration Strategy for LIBS Heavy Metals Analysis in Agriculture Applications
}

\author{
Vasily N. Lednev ${ }^{1, *(\infty)}$, Pavel A. Sdvizhenskii ${ }^{1}{ }^{\circledR}$, Mikhail Y. Grishin ${ }^{1}{ }^{\circledR}$, Evgeny A. Nikitin ${ }^{2}{ }^{\circledR}$, \\ Sergey V. Gudkov ${ }^{1}$ (D) and Sergey M. Pershin ${ }^{1}$ \\ 1 Prokhorov General Physics Institute, Russian Academy of Sciences, 38 Vavilova Street, \\ 119991 Moscow, Russia; sdvizhenskii.pa@misis.ru (P.A.S.); mikhail.grishin@kapella.gpi.ru (M.Y.G.); \\ S_makariy@rambler.ru (S.V.G.); pershin@kapella.gpi.ru (S.M.P.) \\ 2 FSBSI “Federal Scientific Agroengineering Center VIM", 1st Institutsky Proezd 5, 109428 Moscow, Russia; \\ evgeniy.nicks@yandex.ru \\ * Correspondence: lednev@kapella.gpi.ru; Tel.: +74-(99)-5038777876
}

Citation: Lednev, V.N.; Sdvizhenskii, P.A.; Grishin, M.Y.; Nikitin, E.A.; Gudkov, S.V.; Pershin, S.M. Improving Calibration Strategy for LIBS Heavy Metals Analysis in Agriculture Applications. Photonics 2021, 8, 563. https://doi.org/10.3390/ photonics 8120563

Received: 17 November 2021

Accepted: 7 December 2021

Published: 9 December 2021

Publisher's Note: MDPI stays neutral with regard to jurisdictional claims in published maps and institutional affiliations.

Copyright: (c) 2021 by the authors. Licensee MDPI, Basel, Switzerland. This article is an open access article distributed under the terms and conditions of the Creative Commons Attribution (CC BY) license (https:/ / creativecommons.org/licenses/by/ $4.0 /)$.

\begin{abstract}
A new calibration procedure, known as mapping conditional-calibration laser-induced breakdown spectroscopy (LIBS), has been suggested to improve analysis results for heterogeneous samples. The procedure is based on LIBS elemental mapping, followed by signal conditioning in every sampling spot to skip signal outliers, then by finalizing the calibration curve construction. The suggested mapping conditional calibration procedure was verified for zinc analysis in soybean grist samples. The laser parameters correspond to those of the hand-held LIBS instrument in order to estimate the influence of sample surface heterogeneity under on-site analysis conditions. The laser spot $(60 \mu \mathrm{m})$ was equal to or smaller than the typical size of grist particles $(40-500 \mu \mathrm{m})$ but laser crater dimensions were significantly greater and varied widely $(150-450 \mu \mathrm{m})$. The LIBS mapping of different spectral signals (atomic and ionic lines for major and minor components) was achieved. Elemental maps were normalized to achieve signal maps that were conditionally spotted to skip signal outliers. It was demonstrated that the suggested mapping conditional-calibration LIBS provided $15 \mathrm{ppm}$ RMSECV for zinc determination in heterogeneous samples, which is typical for agricultural products.
\end{abstract}

Keywords: laser-induced breakdown spectroscopy; express analysis; heavy metals; grist quality; cattle food diagnostics

\section{Introduction}

The control of concentrations of major and minor elements in agriculture has been of great interest during the last decade, due to requirements for the online management of nutrients and preventing contamination from toxic metal. The express detection of harmful elements in fresh plants, vegetables, or fruits can enable effective control in the food industry, resulting in safe and healthy food alimentation. Toxic elements and heavy metals in agricultural products can induce intoxication and even diseases, so express multi-elemental analysis techniques are required. Conventional laboratory techniques, such as inductively coupled plasma-atomic emission spectrometry (ICP-AES), inductively coupled plasma mass spectrometry (ICP-MS), graphite furnace atomic absorption spectrometry (GFAAS), flame atomic absorption spectrometry (FAAS), and X-ray fluorescence spectrometry (XRF) have been successfully adopted for food and agricultural products [1-7]. All the abovementioned techniques require laboratory conditions, are time-consuming, and need sample preparation. However, high-quality agricultural production needs instruments that can provide onsite or inline control of heavy elements in plant or food materials, for example, in cattle.

Laser-induced breakdown spectroscopy (LIBS) is of growing interest in agriculture and food production in recent years, due to its capability of express multi-elemental analysis without any sample preparation [8-20]. LIBS was successfully utilized for the analysis 
of different food products like wheat [21,22], vegetables [23,24], milk powder [25-28], bread [29], oil [30] and wine [31]. For example, Beldjilali et al. [24,32] analyzed fresh potato flesh and skin without any sample preparation. They achieved good agreement between LIBS results and the values measured by conventional techniques (ICP-AES, ICP-MS and AAS) for both major and minor elements (Al, Mn, Ti, Li, and $\mathrm{Si}$ ). Later, the same team suggested a two-step procedure for trace element analysis in food utilizing calibration-free LIBS [33].

Three different approaches are used in LIBS to quantify the elemental composition of organic materials [11,32,34]: (a) single or multiple variable calibrations with the standard samples; (b) calibration-free LIBS; (c) chemometrics. Calibration-free LIBS can perform analysis without any reference materials, but such an approach can provide reliable results only for major components, while errors of determination for additives are too large for meaningful analysis [35]. Chemometrics methods became very popular in LIBS within the last decade for both identification and quantitative analysis [36,37]. However, conventional calibration curve construction by reference materials is also widely used, due to its simplicity and good analytical performance [11].

Food and plant samples demonstrate complex composition and the heterogeneous distribution of elements, so LIBS calibration curve construction will be affected by large measurement errors. Because of the samples' complex structure, every food item gives a different response to laser ablation due to differences in absorption coefficients, thermal diffusivity, oil/water concentration, etc. In order to improve LIBS measurements' reproducibility and to achieve a flat surface on the sample, food or plant materials are pressed into tablets $[11,19,38,39]$. The granular sizes in plant material varied from a few microns up to a few millimeters, while an LIBS sampling spot is typically in the $10-200 \mu \mathrm{m}$ range. In order to improve analysis reproducibility, different spots are ablated and the LIBS spectra are quantified and averaged across the sampling area. Typically, internal normalization on the matrix component is utilized to eliminate any unwanted shot-to-shot LIBS signal fluctuations. In such a case, LIBS spectra averaging is a poor choice since the variation of plasma properties caused by the heterogeneity of elements is diminished, so calibration curves will provide poorer analysis results compared to those samples with a homogeneous composition.

In the present paper, we suggest an alternative way of LIBS calibration to account for sample surface heterogeneity. The sampling procedure is based on LIBS elemental mapping and signals leveling in order to avoid large fluctuations of plasma properties during pulseto-pulse measurements, so the calibration curve is constructed with reproducible spectra. Owing to the rapid progress in developing commercial hand-held LIBS analyzers [16,40], it would be most appropriate to test our mapping sampling strategy for the analysis of agriculture samples with such portable devices. Soybean grist samples were analyzed by LIBS, since such material is of high importance in the cattle industry and heavy metal concentrations must be controlled to assure high-quality milk products. Cattle for highquality dairy products need a diet with high fat or protein contents, such as sunflower meal, soybeans, rapeseed, etc., which are the processed products of oilseed processing. In order to increase production efficiency, oil producers will utilize chemical extraction so the processed products (grist) may be contaminated by harmful substances. For this reason, the onsite/inline analysis of cattle food is of high importance.

\section{Experimental Technique}

\subsection{Laser-Induced Breakdown Spectroscopy Setup}

The experiments were carried out at a LIBS setup previously described in detail in [41] and will be described in this study hereafter (Figure 1). Laser parameters were set close to those for hand-held LIBS analyzers (1064 nm, $10 \mathrm{~ns}, 5 \mathrm{~mJ}, 5 \mathrm{~Hz}$, TEM 00, $\varnothing 3 \mathrm{~mm}$ ) in order to achieve the same conditions (power density, sampling spot size). The laser beam was focused by a quartz lens $(\mathrm{F}=110 \mathrm{~mm})$ placed orthogonally to the sample surface on the spot of $60 \mu \mathrm{m}$. Plasma emission was collected by a quartz lens $(\mathrm{F}=60 \mathrm{~mm})$ placed at two focal 
lengths, then the plasma image was transferred at 1:1 magnification to the spectrograph input slit. The spectrograph (Shamrock 303i, Andor, Belfast, UK) was equipped with a gated camera (iStar, Andor, UK) to capture the plasma spectra. The sample holder was installed on a two-dimensional motorized stage (8MT173-30, Standa Ltd., Vilnius, Lithuania) that can be moved in a $25 \mathrm{~mm}$ range, with $0.2 \mu \mathrm{m}$ position precision. Control and synchronization of the motorized stage, laser, and spectrometer were performed via custom-written software developed in the LabVIEW environment. Spectral data processing was also performed via custom-made LabVIEW programs. Here, we utilized a laser with parameters similar to those of hand-held LIBS systems.

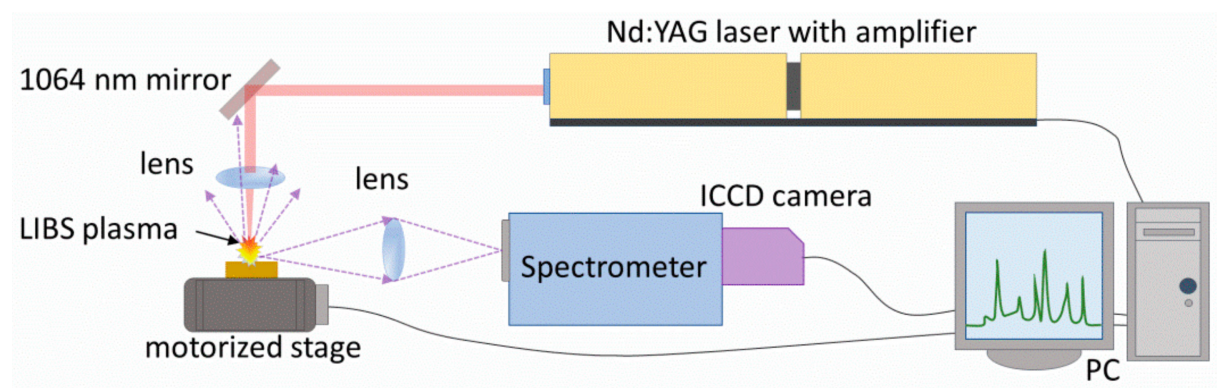

Figure 1. Experiment setup scheme for grist tablet analysis by laser-induced breakdown spectroscopy (LIBS). ICCD—intensified charge-coupled device camera, PC—personal computer.

\subsection{Samples}

Commercially available soybean grist was purchased from Rusagro LLC (Tambov, Russia). The grist was milled in a milling machine (JSM 100, Herzeg, Nattheim, Germany) for $5 \mathrm{~min}$ and then the material was pressed into pellets under a load of $150 \mathrm{kN}$ (Retsch PP25, Haan, Germany). A photograph of the grist pellets can be seen in Figure 2. After creating the pellets, they were analyzed with an X-ray fluorescence spectrometer (ARL 9900, Thermo Fisher Scientific, New York, NY, USA) [42], which revealed a low concentration of Zn (Table 1).
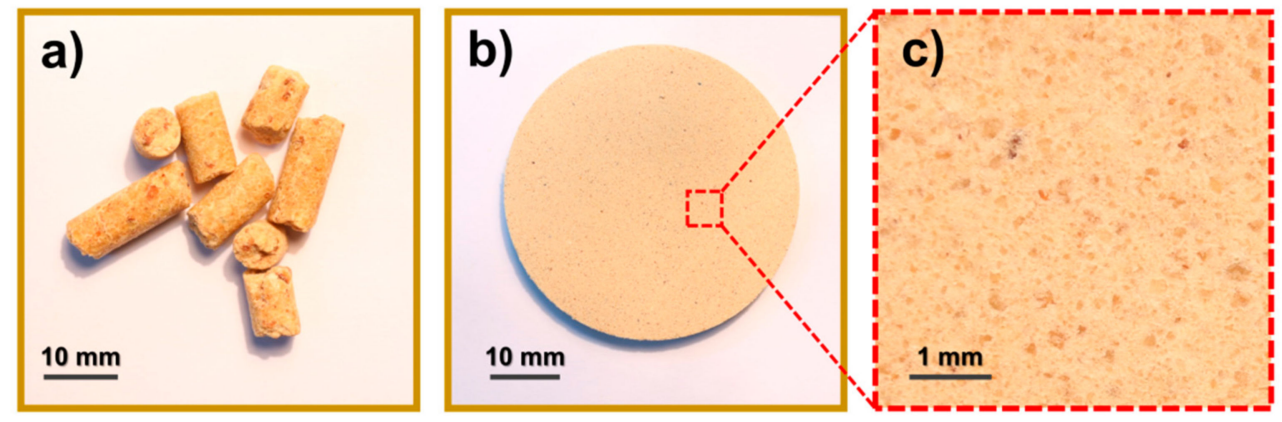

Figure 2. Soybean grist samples_-color photo (a), milled and pressed soybean tablet photo (b), and microscopy image of the tablet surface (c).

Table 1. Soybean grist elemental composition (wt. \%) as revealed by X-ray fluorescence spectrometry (ARL 9900, Thermo Fisher Scientific).

\begin{tabular}{lcccccc}
\hline \multicolumn{1}{c}{ Sample } & $\mathbf{K}$ & $\mathbf{C a}$ & $\mathbf{M g}$ & $\mathbf{Z n}$ & $\mathbf{N a}$ & $\mathbf{P b}$ \\
\hline sample1 & 1.67 & 1.0500 & 0.0849 & 0.0645 & 0.0058 & 0.00487 \\
\hline sample2 & 1.70 & 1.0600 & 0.0812 & 0.0395 & 0.0057 & 0.00260 \\
\hline sample3 & 1.69 & 1.0600 & 0.0820 & 0.0520 & 0.0051 & 0.00387 \\
\hline sample4 & 1.68 & 1.0300 & 0.0822 & 0.0270 & 0.0047 & 0.00103 \\
\hline sample5 & 1.72 & 1.0400 & 0.0792 & 0.0195 & 0.0049 & 0.0061 \\
\hline sample6 (raw material) & 1.71 & 1.0500 & 0.0826 & 0.0170 & 0.0059 & $<0.0002$ \\
\hline
\end{tabular}


In order to calibrate the LIBS setup and estimate its analytical capabilities, a series of samples were prepared. The raw grist powder was dried in air heated to $99{ }^{\circ} \mathrm{C}$ for $1 \mathrm{~h}$. The required amount of zinc and lead solutions at known concentrations had been added to the grist powder and then dried in air at $99^{\circ} \mathrm{C}$ for $1 \mathrm{~h}$. Different ratios of raw and "contaminated" grist powders were mixed to get 5 different samples. In order to obtain the uniform distribution of $\mathrm{Zn}$ and $\mathrm{Pb}$ additives, the prepared mixture was blended in an agate mortar for $30 \mathrm{~min}$; it was then dried at $99^{\circ} \mathrm{C}$ for $1 \mathrm{~h}$ to remove any non-bonded water. The obtained powders were pressed into tablets ( $40 \mathrm{~mm}$ diameter, $5 \mathrm{~mm}$ thickness) in air for 5 min under a load of $150 \mathrm{kN}$ (Retsch PP25). The prepared tablets were analyzed with an X-ray fluorescence spectrometer (ARL 9900, Thermo Fisher Scientific). The tablet surface was examined using optical microscopy (Figure 2) to estimate sample non-homogeneity. The samples were fairly heterogeneous and different color particles had dimensions from 20 to $300 \mu \mathrm{m}$; this was equal to or smaller than the $60 \mu \mathrm{m}$ diameter of the laser spot.

\section{Results}

The express elemental analysis of agriculture samples is necessary for modern farming, and hand-held LIBS systems can provide a good solution [16]. Such instruments are based on low-pulse-energy nanosecond lasers $(1-10 \mathrm{~mJ} /$ pulse) that produce a small sampling spot of 30-100 $\mu \mathrm{m}$ in diameter. Typically, several spots are ablated to improve the sample's representative analysis, but the LIBS spectrum is averaged during this measurement. In this study, we reproduced the same conditions for laser ablation and studied in detail the analytical capabilities of LIBS for heavy metals analysis.

The sample was mapped by the LIBS system in the following way: the sample was moved, stopped, and then ablated using three laser pulses. Afterward, the translation stage was further moved to reveal a fresh sample surface for the next LIBS measurement. A series of 900 spots ( $30 \times 30$-spot grid) was ablated. The laser crater dimensions (diameter, depth) and shapes varied widely for different spots, as can be clearly observed by optical microscopy (Figure 3). Large variations in crater dimensions can be explained by the variation of particle absorption coefficients, as well as variations in particle size and bonding at nearby laser spots and nearby areas. For particles with a greater absorption coefficient (dark particles), the ablation efficiency will be greater; thus, plasma temperature and density will be greater, resulting in a higher intensity of lines in the spectrum. The expanding plasma plume will induce a shockwave, followed by a sound wave that will blow away powder particles, so the laser crater diameter will be much greater compared to that for a metal target [43]. The crater diameters varied from 150 to $450 \mu \mathrm{m}$, while the laser spot was only $60 \mu \mathrm{m}$ in diameter.

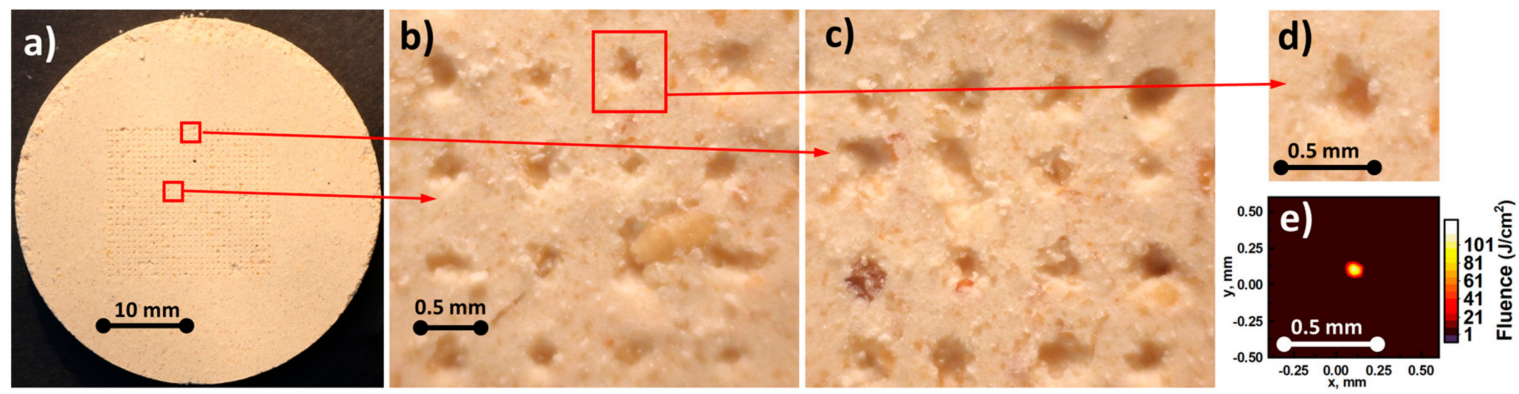

Figure 3. Photograph of the soybean grist tablet surface after LIBS mapping (a), and a more detailed view of the craters (b,c), single laser crater photo (d), and laser beam spot (e).

The optimal choice of LIBS spectral lines depends on a number of factors, including detector sensitivity, line intensity, the possibility of self-absorption, and spectral interference. Additionally, it is conventional to use an internal standardization procedure to compensate for pulse-to-pulse variations in the amount of ablated matter and in the excitation characteristics of plasma. In this study, we focused on zinc analysis at a low concentration, so we needed the strongest lines but not to spectrally interfere with the atomic/ionic lines 
of major elements or molecular bands; for this reason, we chose the spectral region at 200-220 nm, where resonant ionic and atomic lines are present: Zn II at 202.55, Zn II at 206.20, and Zn I at $213.86 \mathrm{~nm}$. Additionally, calcium Ca II 210.32, Ca II 211.28, and phosphorus P I 213.62, P I 214.91 lines were observed in the spectrum. The most intensive zinc line Zn II $213.85 \mathrm{~nm}$ cannot be used as a signal (spectral line integral corrected for background) due to spectral interference with the P I $213.62 \mathrm{~nm}$ line. The evolution of laser-induced plasma spectra has been studied to reveal the optimal conditions for spectra acquisition. Plasma emissivity decreased rapidly within the first five microseconds (Figure 4), so the early moments of plasma emission were optimal for LIBS spectra acquisition (gate 1 us, delay 0.2 us) due to a better signal-to-noise ratio for $\mathrm{Zn}$ lines.
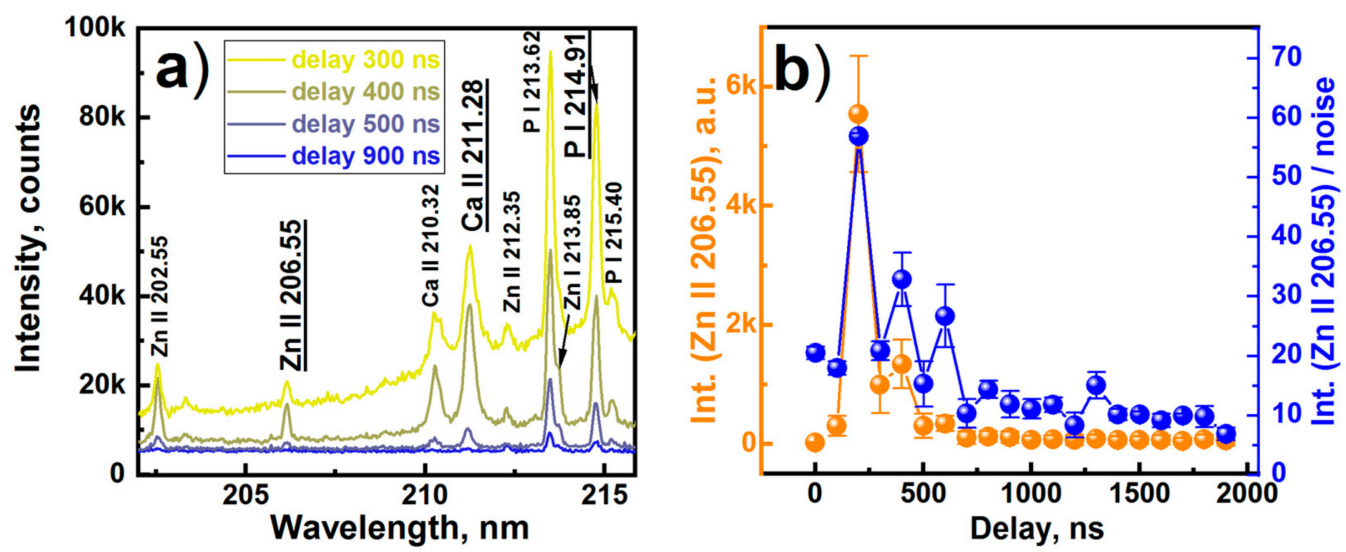

Figure 4. Laser plasma spectra at different moments in plasma evolution (a), the zinc (Zn II) $206.55 \mathrm{~nm}$ line and signal, and the signal-to-noise ratio at different moments of LIBS plasma expansion (b).

In order to provide representative LIBS analysis for a nonhomogeneous target, the sampling area has to be significantly greater compared to the typical particle size. To optimize the time required for a single LIBS measurement and to estimate the minimum sampling area for representative analysis, we have mapped the soybean grist tablets and have constructed maps for major elements (calcium, phosphorus) and zinc (Figure 5). The zinc distribution was fairly uniform, proving proper sample preparation (see Section 2.2); however, some spots with a higher signal can be traced in the map. The same distribution was observed for calcium and phosphorus. The continuous plasma emission (background) was also quantified and mapped since this signal can be used for analytical line normalization [44]. The corresponding maps of Zn II $206.55 \mathrm{~nm}$, normalized on calcium, phosphorus and background signals, are presented in Figure 6.

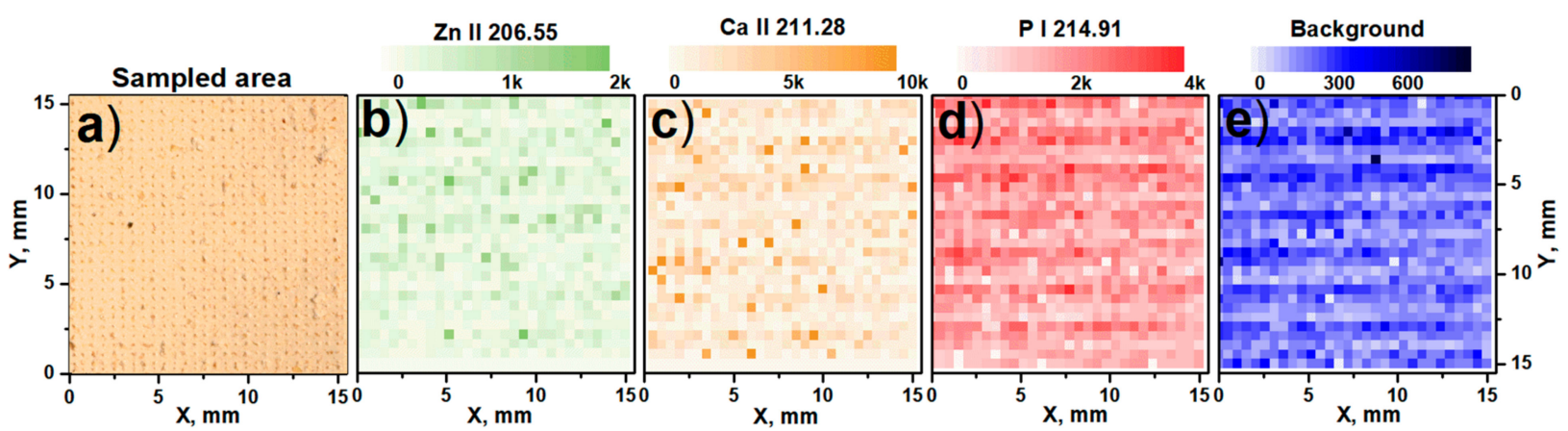

Figure 5. Sample surface area photo after LIBS mapping (a) and corresponding atomic/ionic lines intensity maps for Zn II 206.55 (b), Ca II 211.28 (c), P I 214.91 (d) nm lines, and the plasma emission background (e). 


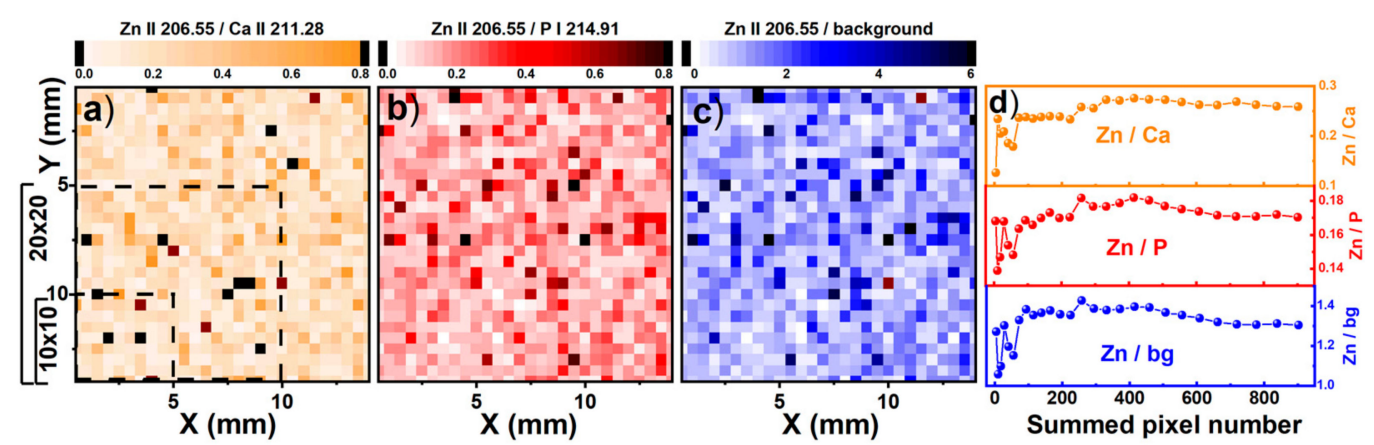

Figure 6. Zinc intensity (Zn II $206.55 \mathrm{~nm}$ ) maps normalized on calcium Ca II $211.28 \mathrm{~nm}$ (a) and phosphorus P I $206.55 \mathrm{~nm}$ (b) lines and background intensity (c). (d)-Normalized zinc intensity, averaged by increasing area dimensions or increased sampling spot number (e.g., $10 \times 10$ square $=100$ spots).

In order to estimate the minimal sampling area needed for representative analysis, we have totaled the points in Figure $6 \mathrm{a}-\mathrm{c}$ to achieve squared areas of different dimensions. Normalized signals had poor reproducibility but, after averaging over 300-400 pulses, the signal stabilized (Figure 6d). A better-normalized signal convergence was determined when normalizing on Ca II $211.28 \mathrm{~nm}$, so it was convenient to use the Zn II $206.55 \mathrm{~nm} / \mathrm{Ca}$ II $211.28 \mathrm{~nm}$ ratio for the construction of the calibration curve. To study this in detail, we have drawn normal box graphs for the Zn II $206.55 \mathrm{~nm}$ line intensity, as well as the signal normalized by phosphor, calcium lines and background level intensity. In order to compare the distribution function of normalized signals, we have also normalized such data by median normalization. According to Figure 7, the narrowest distribution was achieved when the normalization on the Ca II $211.28 \mathrm{~nm}$ line was chosen. Additionally, all normalized signal values beyond $2^{*}$ sigma were considered as outliers, so data were conditioned before the calibration curve construction step.

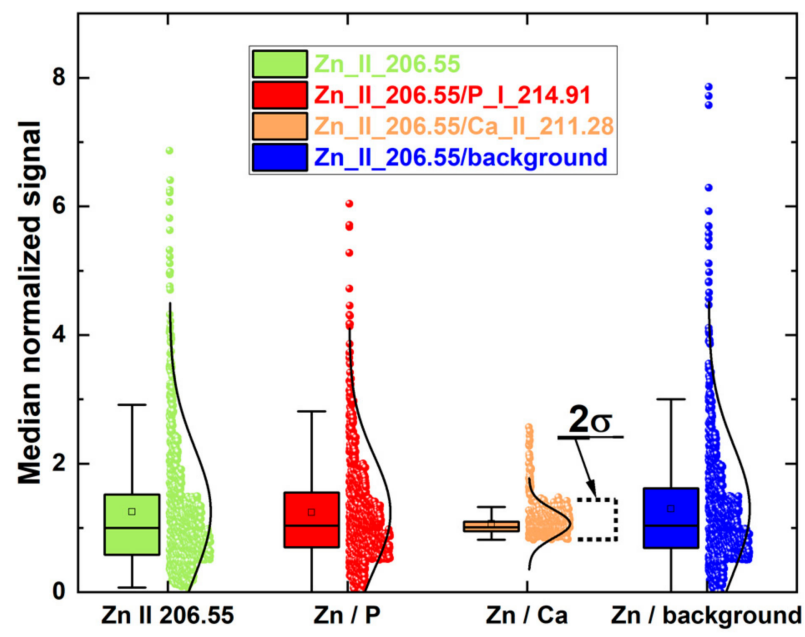

Figure 7. Distributions for median normalized signals for LIBS maps: Zn II $206.55 \mathrm{~nm}$ (green), Zn II 206.55/P I 214.91 (red), Zn II 206.55/Ca II 211.28 (brown), and Zn II 206.55 nm/background (blue). The $\mathrm{Zn} / \mathrm{Ca}$ ratio covered by $2 *$ sigma was taken for calibration curve construction.

Calibration curves for zinc with a different number of averaging spots have been constructed and are presented in Figure 8a. The calibration curves were fitted by linear functions. For viewability, only a few of the calibration curves are shown. LIBS analytical capabilities were compared in terms of precision, calibration curve linearity and analytical accuracy. The accuracy of the analysis was estimated by the leave-one-out cross-validation procedure, with the root mean square error of cross-validation (RMSECV) as the main index, 
estimating the calibration model performance: $\operatorname{RMSECV}=\left(\frac{1}{n} \sum_{i}^{n}\left(c_{i}-c_{i}^{r e f}\right)^{2}\right)^{1 / 2}$, where $c_{i}$-predicted concentration, $c_{i}{ }^{\text {ref }}$-reference concentration, and $n$-number of calibration samples.
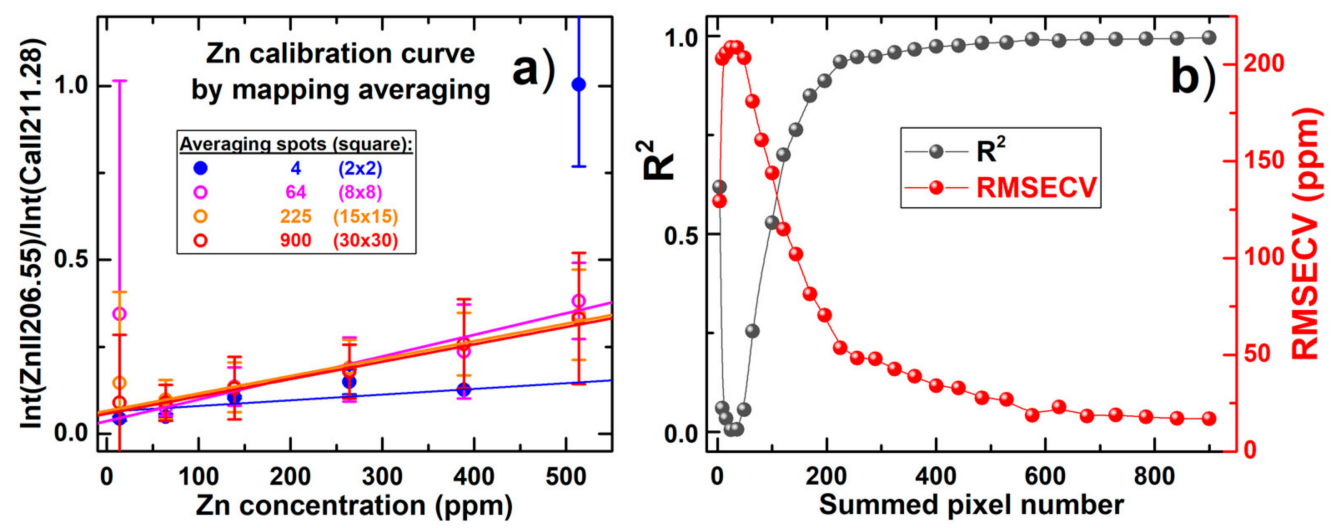

Figure 8. (a)-Calibration curves from mapping the conditional calibration LIBS for a different number of sampling spots, averaging 4-900 spots. (b) - Coefficient of determination $\left(\mathrm{R}^{2}\right)$ and the root mean square error of cross-validation (RMSECV) for regressions as functions of sampling area averaging.

For small-sized sampling areas (up to 225 points), the calibration curve cannot be established for meaningful LIBS analysis (Figure 8a): RMSECV values were in the 50-200 ppm concentration range, which was comparable to the concentration in the reference samples. Starting from 200-300 LIBS sampling spots, a good calibration curve can be achieved; however, additional data points in the calibration will further improve the analysis results. The coefficient of determination $\left(\mathrm{R}^{2}\right.$ factor) was systematically poorer for low-spots averaging. For 250 -spot sampling $\left(15 \times 15\right.$ spots square), the $\mathrm{R}^{2}$ reached 0.936 , so meaningful calibration can be established, but 900 spot-averaging provided a value of $R^{2}=0.996$. The RMSECV value (Figure 8b) showed the same tendency, and after averaging for 300 spots, the accuracy of analysis was in the 15-20 ppm range. It should be noted that hand-held LIBS analyzers should be used with caution for heavy metals analysis in the heterogeneous samples (like grist) since such devices can only automatically map small areas (max spot of $8 \times 8$ ) and thus could not provide a representative sampling.

\section{Conclusions}

Here, we have presented a new approach for the analysis of heterogeneous samples by LIBS - mapping conditional calibration. The procedure is based on the LIBS elemental mapping of large areas, followed by signal conditioning to construct calibration curves. The suggested mapping conditional calibration procedure will be of great interest for the onsite analysis of heterogeneous samples, like the soybean grist pellets utilized in agriculture for cattle nutrition. Hand-held LIBS instruments are widely employed in farming due to their simplicity and the possibility of express analysis, so mapping conditional calibration LIBS was verified using the laser-sampling parameters that are typical for such compact devices (a laser spot diameter of $60 \mu \mathrm{m}$ was equal to or smaller than the typical size of grist particles, 40-500 $\mu \mathrm{m})$. Elemental maps for major components were obtained and normalized, while the signal was conditionally chosen to skip the signal outliers. It was demonstrated for zinc analysis in soybean grist that the suggested mapping conditional calibration LIBS provided an accuracy of determination of 15 ppm. Furthermore, employing LIBS with the mapping conditional calibration procedure will improve the results of an analysis in the case of homogeneous samples, since pulse-to-pulse plasma fluctuations can be traced. 
Author Contributions: V.N.L., conceptualization, investigation, writing-original draft preparation; P.A.S., investigation, formal analysis, visualization; M.Y.G., visualization and language check; E.A.N., resources; S.V.G., supervision, writing-review and editing; S.M.P., writing-review and editing. All authors have read and agreed to the published version of the manuscript.

Funding: The investigation was funded by the Ministry of Science and Higher Education of the Russian Federation for large scientific projects in priority areas of scientific and technological development (contract no. 075-15-2020-774).

Institutional Review Board Statement: Not applicable.

Informed Consent Statement: Not applicable.

Data Availability Statement: Not applicable.

Conflicts of Interest: The authors declare no conflict of interest.

\section{References}

1. Alonso, J.I.G.; Marchante-Gayón, J.M.; Moldovan, M. New developments in food analysis by ICP-MS. In Handbook of Mineral Elements in Food; de la Guardia, M., Garrigues, S., Eds.; Wlley Blackwell: West Sussex, UK, 2015; pp. 239-262. [CrossRef]

2. Marcinkowska, M.; Barałkiewicz, D. Multielemental speciation analysis by advanced hyphenated technique-HPLC/ICP-MS: A review. Talanta 2016, 161, 177-204. [CrossRef]

3. Aceto, M.; Abollino, O.; Bruzzoniti, M.C.; Mentasti, E.; Sarzanini, C.; Malandrino, M. Determination of metals in wine with atomic spectroscopy (flame-AAS, GF-AAS and ICP-AES); a review. Food Addit. Contam. 2002, 19, 126-133. [CrossRef]

4. Katerinopoulou, K.; Kontogeorgos, A.; Salmas, C.E.; Patakas, A.; Ladavos, A. Geographical Origin Authentication of Agri-Food Products: A Review. Foods 2020, 9, 489. [CrossRef] [PubMed]

5. Byers, H.L.; McHenry, L.J.; Grundl, T.J. XRF techniques to quantify heavy metals in vegetables at low detection limits. Food Chem. $X$ 2019, 1, 100001. [CrossRef]

6. Soodan, R.K.; Pakade, Y.B.; Nagpal, A.; Katnoria, J.K. Analytical techniques for estimation of heavy metals in soil ecosystem: A tabulated review. Talanta 2014, 125, 405-410. [CrossRef]

7. Bueno Guerra, M.B.; Adame, A.; de Almeida, E.; Arantes de Carvalho, G.G.; Stolf Brasil, M.A.; Santos, D., Jr.; Krug, F.J. Direct analysis of plant leaves by EDXRF and LIBS: Microsampling strategies and cross-validation. J. Anal. At. Spectrom. 2015, 30, 1646-1654. [CrossRef]

8. Hahn, D.W.; Omenetto, N. Laser-Induced Breakdown Spectroscopy (LIBS), Part II: Review of Instrumental and Methodological Approaches to Material Analysis and Applications to Different Fields. Appl. Spectrosc. 2012, 66, 347-419. [CrossRef] [PubMed]

9. Harmon, R.S.; Russo, R.E.; Hark, R.R. Applications of laser-induced breakdown spectroscopy for geochemical and environmental analysis: A comprehensive review. Spectrochim. Acta B At. Spectrosc. 2013, 87, 11-26. [CrossRef]

10. Markiewicz-Keszycka, M.; Cama-Moncunill, X.; Casado-Gavalda, M.P.; Dixit, Y.; Cama-Moncunill, R.; Cullen, P.J.; Sullivan, C. Laser-induced breakdown spectroscopy (LIBS) for food analysis: A review. Trends Food Sci. Technol. 2017, 65, 80-93. [CrossRef]

11. Sezer, B.; Bilge, G.; Boyaci, I.H. Capabilities and limitations of LIBS in food analysis. TrAC Trends Anal. Chem. 2017, 97, 345-353. [CrossRef]

12. Lednev, V.N.; Sdvizhenskii, P.A.; Grishin, M.Y.; Stavertiy, A.Y.; Tretyakov, R.S.; Asyutin, R.D.; Pershina, S.M. Laser Welding Spot Diagnostics by Laser-Induced Breakdown Spectrometry. Phys. Wave Phenom. 2021, 29, 221-228. [CrossRef]

13. Galbács, G. A critical review of recent progress in analytical laser-induced breakdown spectroscopy. Anal. Bioanal. Chem. 2015, 407, 7537-7562. [CrossRef] [PubMed]

14. Crocombe, R. Portable Spectroscopy. Appl. Spectrosc. 2018, 72, 1701-1751. [CrossRef] [PubMed]

15. Gaudiuso, R.; Melikechi, N.; Abdel-Salam, Z.A.; Harith, M.A.; Palleschi, V.; Motto-Ros, V.; Busser, B. Laser-induced breakdown spectroscopy for human and animal health: A review. Spectrochim. Acta B At. Spectrosc. 2019, 152, 123-148. [CrossRef]

16. Senesi, G.S.; Harmon, R.S.; Hark, R.R. Field-portable and handheld laser-induced breakdown spectroscopy: Historical review, current status and future prospects. Spectrochim. Acta B At. Spectrosc. 2021, 175, 106013. [CrossRef]

17. Pershin, S.M.; Lednev, V.N.; Bunkin, A.F. Laser ablation of alloys: Selective evaporation model. Phys. Wave Phenom. 2011, 19, 261-274. [CrossRef]

18. Elmasry, G.; Kamruzzaman, M.; Sun, D.-W.; Allen, P. Principles and Applications of Hyperspectral Imaging in Quality Evaluation of Agro-Food Products: A Review. Crit. Rev. Food Sci. Nutr. 2012, 52, 999-1023. [CrossRef]

19. Santos, D.; Nunes, L.C.; de Carvalho, G.G.A.; Gomes, M.D.S.; de Souza, P.F.; Leme, F.D.O.; dos Santos, L.G.C.; Krug, F.J. Laser-induced breakdown spectroscopy for analysis of plant materials: A review. Spectrochim. Acta B At. Spectrosc. 2012, 71, 3-13. [CrossRef]

20. Peng, J.; Liu, F.; Zhou, F.; Song, K.; Zhang, C.; Ye, L.; He, Y. Challenging applications for multi-element analysis by laser-induced breakdown spectroscopy in agriculture: A review. TrAC Trends Anal. Chem. 2016, 85, 260-272. [CrossRef]

21. Peruchi, L.C.; Nunes, L.C.; de Carvalho, G.G.A.; Guerra, M.B.B.; de Almeida, E.; Rufini, I.A.; Santos, D.; Krug, F.J. Determination of inorganic nutrients in wheat flour by laser-induced breakdown spectroscopy and energy dispersive X-ray fluorescence spectrometry. Spectrochim. Acta B At. Spectrosc. 2014, 100, 129-136. [CrossRef] 
22. Bilge, G.; Sezer, B.; Eseller, K.E.; Berberoğlu, H.; Köksel, H.; Boyacı, İ.H. Determination of Ca addition to the wheat flour by using laser-induced breakdown spectroscopy (LIBS). Eur. Food Res. Technol. 2016, 242, 1685-1692. [CrossRef]

23. Juvé, V.; Portelli, R.; Boueri, M.; Baudelet, M.; Yu, J. Space-resolved analysis of trace elements in fresh vegetables using ultraviolet nanosecond laser-induced breakdown spectroscopy. Spectrochim. Acta B At. Spectrosc. 2008, 63, 1047-1053. [CrossRef]

24. Beldjilali, S.; Yip, W.L.; Hermann, J.; Baba-Hamed, T.; Belasri, A. Investigation of plasmas produced by laser ablation using single and double pulses for food analysis demonstrated by probing potato skins. Anal. Bioanal. Chem. 2011, 400, 2173. [CrossRef] [PubMed]

25. Lei, W.Q.; El Haddad, J.; Motto-Ros, V.; Gilon-Delepine, N.; Stankova, A.; Ma, Q.L.; Bai, X.S.; Zheng, L.J.; Zeng, H.P.; Yu, J. Comparative measurements of mineral elements in milk powders with laser-induced breakdown spectroscopy and inductively coupled plasma atomic emission spectroscopy. Anal. Bioanal. Chem. 2011, 400, 3303-3313. [CrossRef] [PubMed]

26. Abdel-Salam, Z.; Al Sharnoubi, J.; Harith, M.A. Qualitative evaluation of maternal milk and commercial infant formulas via LIBS. Talanta 2013, 115, 422-426. [CrossRef] [PubMed]

27. Zhao, M.; Markiewicz-Keszycka, M.; Beattie, R.J.; Casado-Gavalda, M.P.; Cama-Moncunill, X.; O’Donnell, C.P.; Cullen, P.J.; Sullivan, C. Quantification of calcium in infant formula using laser-induced breakdown spectroscopy (LIBS), Fourier transform mid-infrared (FT-IR) and Raman spectroscopy combined with chemometrics including data fusion. Food Chem. 2020, 320, 126639. [CrossRef] [PubMed]

28. Moncayo, S.; Manzoor, S.; Rosales, J.D.; Anzano, J.; Caceres, J.O. Qualitative and quantitative analysis of milk for the detection of adulteration by Laser Induced Breakdown Spectroscopy (LIBS). Food Chem. 2017, 232, 322-328. [CrossRef]

29. Bilge, G.; Boyacı, İ.H.; Eseller, K.E.; Tamer, U.; Çakır, S. Analysis of bakery products by laser-induced breakdown spectroscopy. Food Chem. 2015, 181, 186-190. [CrossRef]

30. Mbesse Kongbonga, Y.G.; Ghalila, H.; Onana, M.B.; Ben Lakhdar, Z. Classification of vegetable oils based on their concentration of saturated fatty acids using laser induced breakdown spectroscopy (LIBS). Food Chem. 2014, 147, 327-331. [CrossRef]

31. Moncayo, S.; Rosales, J.D.; Izquierdo-Hornillos, R.; Anzano, J.; Caceres, J.O. Classification of red wine based on its protected designation of origin (PDO) using Laser-induced Breakdown Spectroscopy (LIBS). Talanta 2016, 158, 185-191. [CrossRef]

32. Beldjilali, S.; Borivent, D.; Mercadier, L.; Mothe, E.; Clair, G.; Hermann, J. Evaluation of minor element concentrations in potatoes using laser-induced breakdown spectroscopy. Spectrochim. Acta B At. Spectrosc. 2010, 65, 727-733. [CrossRef]

33. Chen, C.-T.; Banaru, D.; Sarnet, T.; Hermann, J. Two-step procedure for trace element analysis in food via calibration-free laser-induced breakdown spectroscopy. Spectrochim. Acta B At. Spectrosc. 2018, 150, 77-85. [CrossRef]

34. Zhang, H.; Wang, S.; Li, D.; Zhang, Y.; Hu, J.; Wang, L. Edible Gelatin Diagnosis Using Laser-Induced Breakdown Spectroscopy and Partial Least Square Assisted Support Vector Machine. Sensors 2019, 19, 4225. [CrossRef] [PubMed]

35. Tognoni, E.; Cristoforetti, G.; Legnaioli, S.; Palleschi, V. Calibration-Free Laser-Induced Breakdown Spectroscopy: State of the art. Spectrochim. Acta B At. Spectrosc. 2010, 65, 1-14. [CrossRef]

36. Kim, G.; Kwak, J.; Choi, J.; Park, K. Detection of Nutrient Elements and Contamination by Pesticides in Spinach and Rice Samples Using Laser-Induced Breakdown Spectroscopy (LIBS). Agric. Food Chem. 2012, 60, 718-724. [CrossRef] [PubMed]

37. Bilge, G.; Velioglu, H.M.; Sezer, B.; Eseller, K.E.; Boyaci, I.H. Identification of meat species by using laser-induced breakdown spectroscopy. Meat Sci. 2016, 119, 118-122. [CrossRef] [PubMed]

38. Nunes, L.C.; Batista Braga, J.W.; Trevizan, L.C.; Florêncio de Souza, P.; Arantes de Carvalho, G.G.; Júnior, D.S.; Poppi, R.J.; Krug, F.J. Optimization and validation of a LIBS method for the determination of macro and micronutrients in sugar cane leaves. J. Anal. At. Spectrom. 2010, 25, 1453-1460. [CrossRef]

39. Galiová, M.; Kaiser, J.; Novotný, K.; Hartl, M.; Kizek, R.; Babula, P. Utilization of laser-assisted analytical methods for monitoring of lead and nutrition elements distribution in fresh and dried Capsicum annuum 1. leaves. Microsc. Res. Tech. 2011, 74, 845-852. [CrossRef] [PubMed]

40. Erler, A.; Riebe, D.; Beitz, T.; Löhmannsröben, H.-G.; Gebbers, R. Soil Nutrient Detection for Precision Agriculture Using Handheld Laser-Induced Breakdown Spectroscopy (LIBS) and Multivariate Regression Methods (PLSR, Lasso and GPR). Sensors 2020, 20, 418. [CrossRef]

41. Lednev, V.; Pershin, S.M.; Bunkin, A.F. Laser beam profile influence on LIBS analytical capabilities: Single vs. multimode beam. J. Anal. At. Spectrom. 2010, 25, 1745. [CrossRef]

42. The Thermo Scientific ARL 9900 Instrument, (n.d.). Available online: https://www.thermofisher.com/order/catalog/product/ IQLAAHGABMFAAJMABT (accessed on 6 December 2021).

43. Gornushkin, S.I.; Gornushkin, I.B.; Anzano, J.M.; Smith, B.W.; Winefordner, J.D. Effective Normalization Technique for Correction of Matrix Effects in Laser-Induced Breakdown Spectroscopy Detection of Magnesium in Powdered Samples. Appl. Spectrosc. 2002, 56, 433-436. [CrossRef]

44. Guezenoc, J.; Gallet-Budynek, A.; Bousquet, B. Critical review and advices on spectral-based normalization methods for LIBS quantitative analysis. Spectrochim. Acta B At. Spectrosc. 2019, 160, 105688. [CrossRef] 